\title{
Two-fluid model for a fluid with tetrahedral-octupolar order
}

\author{
Helmut R. Brand ${ }^{1,2, *}$ and Harald Pleiner ${ }^{2, \dagger}$ \\ ${ }^{1}$ Department of Physics, University of Bayreuth, 95440 Bayreuth, Germany \\ ${ }^{2}$ Max Planck Institute for Polymer Research, 55021 Mainz, Germany
}

(Received 13 May 2021; accepted 5 October 2021; published 26 October 2021)

\begin{abstract}
We investigate the macroscopic dynamics of a two-fluid system with tetrahedral order. As all normal-fluid two-fluid systems one has - compared to a simple fluid — the velocity difference between the two subsystems and the concentration of one component as additional macroscopic variables. Depending on the type of system, the concentration can either be a conserved quantity or relax on a long, but finite timescale. Due to the existence of the tetrahedral order such a system breaks parity symmetry. Here we discuss physical systems without preferred direction in real space, meaning that our description applies to optically isotropic materials. We find a number of reversible as well as dissipative dynamic cross-coupling terms due to the additional octupolar order, when compared to a fluid mixture. As the most interesting cross-coupling term from an experimental point of view, we identify a dissipative cross-coupling between the relative velocity and the usual velocity gradients. Applying a shear flow in a plane, this dissipative cross-coupling leads to a velocity difference perpendicular to the shear plane. As a result one can obtain a spatially homogeneous oscillation of the relative velocity. In addition, this induced relative velocity can couple as a function of time and space to the concentration, which gives rise to an overdamped propagating soundlike mode, where the overdamping arises from the fact that velocity difference is a macroscopic variable and not strictly conserved. We also show that electric field gradients are connected with an analogous reversible cross-coupling and can lead in a planar shear geometry to an overdamped propagating mode as well.
\end{abstract}

DOI: 10.1103/PhysRevE.104.044705

\section{INTRODUCTION}

The hydrodynamics of condensed systems has a long history for the description of macroscopic and mesoscopic phenomena at sufficiently long wavelengths and low frequencies (compare, for example, Refs. [1-3]). It has been applied to many systems including crystals [2], simple fluids and miscible fluid mixtures [1], superfluids [4-6], and liquid crystals [7-9]. To derive hydrodynamics linear irreversible thermodynamics and conservation laws as well as spontaneously broken continuous symmetries are key ingredients [2-7,9].

It became also clear that there is a large class of complex liquids and soft matter materials composed of immiscible subsystems for which also a macroscopic description is desirable. This class includes fluid emulsions [10], colloidal suspensions [11], and polymer solutions and mixtures [12]. A description of such systems that allows for a different dynamics of the subsystems, generally is called a two-fluid description. A systematic derivation of a macroscopic two-fluid description involving mixtures of a simple fluid with nematic liquid crystals and an elastic medium has been presented in Refs. [13,14].

\footnotetext{
*brand@uni-bayreuth.de

†pleiner@mpip-mainz.mpg.de
}

Published by the American Physical Society under the terms of the Creative Commons Attribution 4.0 International license. Further distribution of this work must maintain attribution to the author(s) and the published article's title, journal citation, and DOI. Open access publication funded by the Max Planck Society.
As additional macroscopic variables the velocity difference of the two subsystems is taken into account and, in addition, a concentration variable related to one of the two subsystems. The term macroscopic variables is used here to denote variables that relax on a sufficiently long, but finite timescale. This concept has been introduced by Khalatnikov [4] for the relaxation of the order parameter modulus near the $\lambda$-transition in superfluid ${ }^{4} \mathrm{He}$ and has since been applied to many systems [9].

In this manuscript we only consider the case that the relative velocity is a macroscopic variable in this spirit and relaxes always on a finite timescale. In particular, we do not consider superfluid systems, where, in addition to the normal velocity, also the superfluid velocity is a true hydrodynamic variable due to the spontaneously broken gauge symmetry. The hydrodynamic consequences of this fact have been analyzed for superfluid ${ }^{4} \mathrm{He}[4,5]$ as well as for superfluid ${ }^{3} \mathrm{He}[6$, 15-19] and most recently for the superfluid phases of ${ }^{3} \mathrm{He}$ in a strongly anisotropic aerogel [20].

The concentration can be either a conserved hydrodynamic variable as for colloidal particles in a colloidal suspension or a relaxing macroscopic one, in the case when particles from one subsystem can be converted into another. The latter is the case, for example, for the cluster formation above the nematic-smectic $A$ transition in liquid crystals studied recently in a two-fluid picture [21]. In this manuscript we will consider both cases.

Recently, macroscopic two-fluid descriptions have been given for magneto-rheological fluids (simple liquid carrier fluid and field-responsive magnetic particles) [22], for the 
breakdown of flow alignment in nematic liquid crystals with smectic clusters [21], for elastic composite materials made of two solid compounds [23], as well as for the formation of viscoelastic clusters above the glass transition [24].

In the present paper we present a study of the macroscopic behavior of a two-fluid system in which one of the two fluids is a truly isotropic liquid, while the other one is a tetrahedral system with $T_{d}$ symmetry, a subgroup of the cubic symmetry group [25], that has been introduced to the field of liquid crystals by Fel [26]. In the following years the theoretical investigations involving tetrahedral order focused on phase transitions [26-28] and on many aspects of single-fluid macroscopic dynamics [26,29-32].

Tetrahedral order is described by a rank-3, fully symmetric tensor $T_{i j k}$ that can be represented by the four equivalent position vectors, $n_{i}^{\alpha}, \alpha=1,2,3,4$, of the edges of a regular tetrahedron, $T_{i j k}=\sum_{\alpha=1}^{4} n_{i}^{\alpha} n_{j}^{\alpha} n_{k}^{\alpha}$. It does not contain polar order (due to $T_{i k k} \equiv 0$ ) nor any type of conventional nematic quadrupolar order described by $Q_{i j}$. Indeed, since $T_{i j k}$ is related to the third order (octupolar) mass moment distribution the names tetrahedral and octupolar order are both used.

Since the position vectors are real (polar) vectors, $T_{i j k}$ changes sign under spatial inversion and, thus breaks parity symmetry. Indeed the inverted structure $-T_{i j k}$ differs from the original one by a $\pi / 2$ rotation about one of the fourfold symmetry axis of the tertahedron. Obviously, this kind of parity breaking is quite different from that in polar systems (due to a polar vector) or in chiral systems (due to a pseudoscalar quantity).

The tetrahedral order breaks spatial inversion symmetry, spontaneously. That means, the orientation of $T_{i j k}$ is arbitrary and rigid rotations of $T_{i j k}$ do not cost energy and are therefore slow symmetry variables that have to be included in the list of hydrodynamic variables. This is similar to the director in nematic liquid crystal. However, not all changes of $T_{i j k}$ describe pure rotations - only those that are perpendicular to the original state. They are described by $\delta \Gamma_{i} \equiv(1 / 4 \beta) \epsilon_{i p q} T_{p k l} \delta T_{q k l}$ with a suitable normalization $\beta$. The similarity to nematics is obvious, where not all changes of the director are hydrodynamic variables, but only the perpendicular ones, $\epsilon_{i j k} n_{i} \delta n_{j}$. The connection is even closer to biaxial, orthorhombic nematics [9]—-the latter however do not break parity. It should be noted that the three $\delta \Gamma_{i}$, representing the three Euler angles, do not form a vector, since, e.g., $\delta_{1} \delta_{2} \Gamma_{i} \neq \delta_{2} \delta_{1} \Gamma_{i}$. This causes some additional nonlinear contributions, for details cf. Ref. [32].

A material that only shows tetrahedral order is optically isotropic, since $T_{i j k}$ cannot couple to the dielectric (rank-2) tensor. This makes the direct detection of $T_{d}$ materials rather difficult. However, there are some indications of the presence of a tetrahedral phase in certain bent-core systems in the vicinity of the nematic phase transition [33,34]. In Ref. [33] it was shown that in high magnetic fields a strongly first order-of a magnitude not found for rodlike moleculesphase transition to the nematic phase could be induced and it was suggested that this could be due to tetrahedral order. In Ref. [34] two peaks in the isotropic phase above the nematic phase were found in heat capacity measurements indicating the presence of two distinct optically isotropic phases. Also the magnetic field induced birefringence showed significant qualitative changes compared to rodlike molecules [35], but could be accounted for by a model involving quadrupolar $\left(Q_{i j}\right)$ as well as octupolar $\left(T_{i j k}\right)$ order [34]. There are additional indications for tetrahedral phases involving smectic phases, which are discussed in the review [32].

For various aspects of molecular and microscopic modeling in liquid crystals involving tetrahedral order we refer to Refs. [36-39]. More recently there has also been growing interest in various mathematical aspects of octupolar order in two and three spatial dimensions, in particular by Virga's group [40-42]. We also emphasize that the interest in tetrahedral order goes beyond liquid crystals (and their optically isotropic phases), namely, in the direction of other soft matter systems. As an example we mention applications of octupolar order in the field of movable and deformable active particles in two dimensions by Ohta's group [43-45], which have been designed to model self-propelled micro-organisms.

Here we present a macroscopic two-fluid description of a combined system consisting of a tetrahedral phase (or tetrahedral clusters) embedded in a simple isotropic liquid. It should be noted that our description is based on macroscopic symmetries and does not refer to any specific microscopic model or specific molecules. The total system breaks parity symmetry due the properties of the tetrahedral phase discussed above. It is optically isotropic and therefore difficult to detect. It is the main purpose of this manuscript to analyze, how such a two-fluid system could be identified experimentally due to the presence of the relative velocity (between the two subsystems) as a macroscopic variable.

As we will show a planar shear flow can trigger a relative velocity in the direction perpendicular to the shear plane. This effect will in turn generate further spatiotemporal dynamic effects including an overdamped propagating mode due to the coupling to the concentration variable of one subsystem. We will also briefly outline that similar physically observable effects can arise when planar electric field gradients are applied to an ordered sample of such a two-fluid system. None of the effects predicted in this paper can arise for an optically isotropic one-component system, which is either truly isotropic or purely tetrahedral.

In Sec. II we present the details of the macroscopic dynamics for a two-fluid model with tetrahedral order. In Sec. III we investigate the coupling between relative velocities and velocity gradients for a shear-flow set-up. In Sec. IV we summarize the results and give a perspective. In Appendix A we study the effect of an external extensional flow, in Appendix B we discuss selected dynamic effects of electric fields and their gradients, while in Appendix $\mathrm{C}$ we discuss a reversible coupling of the velocity difference to tetrahedral order in the chiral $T$ phase.

\section{MACROSCOPIC TWO-FLUID DYNAMICS FOR A FLUID WITH TETRAHEDRAL ORDER}

\section{A. Variables}

For the hydrodynamics of any system one has to take into account as variables all locally conserved quantities, e.g., the momentum density $g_{i}$, the mass density $\rho$, and the total energy density $\varepsilon$. For a simple isotropic fluid this is all one needs. On the macroscopic level we describe the present system as 
a homogeneous mixture of a simple fluid with density $\rho_{s}$ and of a fluid with tetrahedral order and density $\rho_{h}$, adding up to the total density $\rho=\rho_{h}+\rho_{s}$. In addition, we allow the two species to move with two distinct velocities, $v_{i}^{s}$ and $v_{i}^{h}$, respectively. They are related to the appropriate single momentum densities $g_{i}^{s}=\rho_{s} v_{i}^{s}$ and $g_{i}^{h}=\rho_{h} v_{i}^{h}$ that add up to the total momentum density $g_{i}=\rho v_{i}$, thereby defining the mean velocity $v_{i}$. For more details of these two-fluid aspects cf. Ref. [13,14].

Compared to a simple fluid we take into account as additional variables the concentration $\phi=\rho_{h} / \rho$ and the relative velocity $w_{i}=v_{i}^{h}-v_{i}^{s}$. In the limit of a single fluid there is $w_{i} \rightarrow 0$ and $\phi \rightarrow 0$. The relative velocity is a slowly relaxing quantity. In this paper we deal with both possibilities for the dynamics of the concentration variable $\phi$, either diffusive or relaxing. The first case applies to situations, like in a binary colloidal mixture, where the constituents of the two fluids are conserved individually requiring material transport for diffusion. A relaxational behavior is possible, when the constituents of the isotropic and the tetrahedral fluid are molecularly the same-just either ordered or not. In the following we discuss the dynamics first for the (more general) relaxational case and discuss the restrictions that apply in the conserved case.

The present composite fluid has in addition tetrahedral order that spontaneously breaks rotational symmetry. This is manifest by the existence of a symmetric rank-3 tensor $T_{i j k}$ that is built by the four position vectors of the corners of a tetrahedron [26]. The orientation of this tetrahedron in space is arbitrary and not energetically fixed, except by external fields or boundaries. Of the ten independent elements of $T_{i j k}$ only three are related to pure, rigid rotations

$$
\delta \Gamma_{i} \equiv(1 / 4 \beta) \epsilon_{i p q} T_{p k l} \delta T_{q k l}
$$

and act therefore as additional hydrodynamic variables. The other elements are related to internal deformations of the tetrahedron and are therefore microscopic nonhydrodynamic variables, e.g., always relax to their equilibrium values on macroscopic timescales. The same also applies to the normalization $\beta$, which is taken as constant. The broken parity symmetry does not give rise to a hydrodynamic variable, since parity changes are not continuous.

The first law of thermodynamics relates changes of all macroscopic variables to changes of the energy density $\varepsilon$ by the Gibbs relation $[2,9,46]$

$$
d \varepsilon=T d \sigma+\mu d \rho+\Pi d \phi+\boldsymbol{v} \cdot d \boldsymbol{g}+\boldsymbol{m} \cdot d \boldsymbol{w}+h_{i}^{\Gamma} d \Gamma_{i} .
$$

The last term $h_{i}^{\Gamma} d \Gamma_{i}$ is a short-hand notation for $\tilde{h}_{i}^{\Gamma} d \Gamma_{i}+$ $\Psi_{i j}^{\Gamma} d \nabla_{j} \Gamma_{i}$ with the effective conjugate tetrahedral field

$$
h_{i}^{\Gamma}=\tilde{h}_{i}^{\Gamma}-\nabla_{j} \Psi_{i j}^{\Gamma}-2 \epsilon_{i k l} \Psi_{k j} \nabla_{j} \Gamma_{l},
$$

where the last contribution is due to the relation $[30,32]$ $\left(\delta_{1} \delta_{2}-\delta_{2} \delta_{1}\right) \Gamma_{i}=2 \epsilon_{i p q}\left(\delta_{1} \Gamma_{p}\right)\left(\delta_{2} \Gamma_{q}\right)$. This relation indicates that $\Gamma_{i}$ does not behave like an ordinary vector in a nonlinear description. The field $\tilde{h}_{i}^{\Gamma}$ is nonzero only in the presence of external orienting forces.

The Gibbs relation contains the entropy density $\sigma$, representing the thermal degree of freedom, with its thermo- dynamic conjugate, the temperature $T$. Other conjugates are the chemical potential $\mu$, the osmotic pressure $\Pi$, the mean velocity $v_{i}=g_{i} / \rho$, and $m_{i}$, the conjugate field to $w_{i}$.

From the requirement that the energy of the system is a first order Eulerian form of all extensive variables, one gets for the pressure $p \equiv-(\partial / \partial V) \int \varepsilon d V=-(\partial / \partial V) E=-\varepsilon+$ $T \sigma+\rho \mu+v_{i} g_{i}$ the Gibbs-Duhem relation

$$
\begin{aligned}
d p= & \sigma d T+\rho d \mu-\Pi d \phi+g \cdot d \boldsymbol{v}-\boldsymbol{m} \cdot d \boldsymbol{w} \\
& -\tilde{h}_{i}^{\Gamma} d \Gamma_{i}-\Psi_{i j}^{\Gamma} d \nabla_{j} \Gamma_{i} .
\end{aligned}
$$

\section{B. Statics}

Hydrodynamics allows us to describe the statics and the dynamics of a system in two separate steps. The statics is given by the relation between the conjugate fields and the variables, and the dynamics relates the time derivatives of the variables to the phenomenological currents, which themselves are expressed by the conjugates or their gradients.

The static behavior of our macroscopic system is conveniently described by the (total) energy density (including the kinetic part)

$$
\begin{aligned}
2 \varepsilon= & \frac{T}{C_{V}}(\delta \sigma)^{2}+\frac{1}{\kappa_{\phi}}(\delta \phi)^{2}+\frac{1}{\rho^{2} \kappa_{\mu}}(\delta \rho)^{2} \\
& +\frac{2}{\alpha_{\phi}}(\delta \phi)(\delta \sigma)+\frac{2}{\rho \alpha_{\rho}}(\delta \rho)(\delta \sigma)+\frac{2}{\rho \kappa_{\pi}}(\delta \rho)(\delta \phi) \\
& +K_{i j k l}^{\Gamma}\left(\nabla_{j} \Gamma_{i}\right)\left(\nabla_{l} \Gamma_{k}\right)+\frac{1}{2 \rho} g^{2}+\rho \phi(1-\phi) \boldsymbol{w}^{2},
\end{aligned}
$$

with

$$
K_{i j k l}^{\Gamma}=K_{1}^{\Gamma}\left(\delta_{i k} \delta_{j l}+\delta_{i l} \delta_{j k}\right)+K_{2}^{\Gamma} \delta_{i k} \delta_{j l}+K_{3}^{\Gamma} T_{j l p} T_{i k p}
$$

describing the (orientational-)elastic energy due to distortions of the tetrahedral orientation [30,32].

From Eq. (5) the relations between variables and conjugate quantities are found by partial derivation and read

$$
\begin{gathered}
\delta T=\frac{T}{C_{V}} \delta \sigma+\frac{1}{\alpha_{\phi}} \delta \phi+\frac{1}{\rho \alpha_{\rho}} \delta \rho, \\
\Pi=\frac{1}{\kappa_{\phi}} \delta \phi+\frac{1}{\rho \kappa_{\pi}} \delta \rho+\frac{1}{\alpha_{\phi}} \delta \sigma+\boldsymbol{w} \cdot \boldsymbol{g} \\
+\rho \boldsymbol{w}^{2}(1-2 \phi), \\
\mu=\frac{1}{\rho^{2} \kappa_{\mu}} \delta \rho+\frac{1}{\rho \kappa_{\pi}} \delta \phi+\frac{1}{\rho \alpha_{\rho}} \delta \sigma+\boldsymbol{w}^{2} \phi(1-\phi), \\
\Psi_{i j}^{\Gamma}=K_{i j k l}^{\Gamma} \nabla_{l} \Gamma_{k}, \\
v_{i}=\frac{1}{\rho} g_{i}, \\
m_{i}=\phi(1-\phi) \rho w_{i} \equiv \alpha w_{i} .
\end{gathered}
$$

The $w_{i}$-dependence of the chemical potential and the osmotic pressure are due to the $\rho$ - and $\phi$-dependence of $\alpha$. 


\section{Dynamic equations}

The full dynamical equations for the tetrahedral rotations $\Gamma_{i}$, the concentration $\phi$, the relative velocity, $w_{i}$, and the fluid degrees of freedom are [13,14,32]

$$
\begin{gathered}
\dot{\varepsilon}+\nabla_{i}(\varepsilon+p) v_{i}+\nabla_{i}\left(j_{i}^{(\varepsilon) R}+j_{i}^{(\varepsilon) D}\right)=0, \\
\dot{\sigma}+\nabla_{i}\left(\sigma v_{i}+j_{i}^{(\sigma) R}+j_{i}^{(\sigma) D}\right)=\frac{2 R}{T}, \\
\dot{\rho}+\nabla_{i}\left(\rho v_{i}\right)=0 \\
\dot{g}_{i}+\nabla_{j}\left(g_{i} v_{j}\right)+\nabla_{i} p+\nabla_{j}\left(\sigma_{i j}^{n l}+\sigma_{i j}^{R}+\sigma_{i j}^{D}\right)=0, \\
\dot{\phi}+v_{j} \nabla_{j} \phi+\frac{1}{\rho} \nabla_{i} m_{i}+I_{\phi}^{R}+I_{\phi}^{D}=0, \\
\dot{w}_{i}+v_{j} \nabla_{j} w_{i}+\nabla_{i}\left(\rho^{-1} \Pi\right)+X_{i}^{R}+X_{i}^{D}=0, \\
\dot{\Gamma}_{i}+v_{j} \nabla_{j} \Gamma_{i}-\frac{1}{2} \epsilon_{i j k} \nabla_{j} v_{k}+Y_{i}^{R}+Y_{i}^{D}=0,
\end{gathered}
$$

with $[13,14]$

$$
\dot{\phi}+v_{j} \nabla_{j} \phi+\frac{1}{\rho} \nabla_{i} m_{i}+\frac{1}{\rho} \nabla_{i}\left(j_{i}^{(h) R}+j_{i}^{(h) D}\right)=0
$$

replacing Eq. (17) in the case of a conserved density $\rho_{h}$ with $\dot{\rho}_{h}+\nabla_{i}\left(\rho_{h} v_{i}^{h}+j_{i}^{(h)}\right)=0$. Note that in Eq. (19) the vorticity term is a linearized version of a more complicated expression [30]. The energy conservation law, Eq. (13), is not independent and therefore redundant due to the Gibbs relation, Eq. (2).

The energy dissipation function $R$ restricts the reversible (superscript $R$ ) and irreversible, dissipative (superscript $D$ ) phenomenological currents. The second law of thermodynamics requires

$$
\begin{aligned}
2 R & =-j_{i}^{(\sigma) *} \nabla_{i} T+I_{\phi}^{*} \Pi-\sigma_{i j}^{*} \nabla_{j} v_{i}+X_{i}^{*} m_{i}+Y_{i}^{*} h_{i}^{\Gamma} \\
& \geqslant 0,
\end{aligned}
$$

with the equal $\operatorname{sign}(>\operatorname{sign})$ for $*=R(*=D)$. In case of conserved densities, $I_{\phi}^{*} \Pi$ is replaced by $-j_{i}^{(h) *} \nabla_{i}(\Pi / \rho)$. From Eq. (21) it follows that the entropy production must be positive for dissipative contributions and zero for reversible contributions. As a result, the diagonal dissipative parameters are positive and for the nondiagonal ones there exist upper bounds, while for reversible parameters either sign is possible and no bounds can be given in general. The explicit conditions for dissipative parameters are given after Eq. (36) while for the reversible coefficients in Eqs. (23)-(28) no such conditions exist.

Equations (13)-(19) contain, apart from the phenomenological currents, also transport and convection whenever appropriate. Those effects are reversible and, indeed, all transport contributions (including the isotropic pressure $p$ and the nonlinear stress $\sigma_{i j}^{n l}$ ) add up to zero entropy production. In particular, $R=0$ requires [32]

$$
2 \sigma_{i j}^{n l}=\Psi_{k j}^{\Gamma} \nabla_{i} \Gamma_{k}+\Psi_{k i}^{\Gamma} \nabla_{j} \Gamma_{k}-\frac{3}{2} \epsilon_{i j k} \nabla_{l} \Psi_{k l}^{\Gamma} .
$$

This form of $\sigma_{i j}^{n l}$ guarantees local angular momentum conservation [2,9].

In the whole set of dynamic equations the mean velocity $v_{i}$ has been chosen as transport and convective velocity for all variables. This allows us to ensure zero entropy production. Due to various material dependent contributions in the reversible currents (see below), the actual convective velocities can be different from $v_{i}$ and can be specific for the different variables.

\section{Reversible currents}

For the reversible parts of the currents in Eqs. (14)-(19) we find, requiring $R=0$ in Eq. (21), the following contributions allowed by spatial and time reversal symmetry:

$$
\begin{gathered}
j_{i}^{(\sigma) R}=\beta_{1} m_{i}+\Gamma_{2} T_{i j k} \nabla_{j} v_{k}+\Gamma_{4} T_{i j k} \nabla_{j} m_{k}, \\
\sigma_{i j}^{R}=2 \beta_{2} m_{i} w_{j}+\beta_{7} \delta_{i j} \Pi-\Gamma_{2} T_{i j k} \nabla_{k} T \\
\quad-\Gamma_{3} T_{i j k} \nabla_{k}(\Pi / \rho) \\
X_{i}^{R}=\beta_{1} \nabla_{i} T+\gamma \nabla_{i} \Pi+\beta_{2} w_{j}\left(\nabla_{i} v_{j}+\nabla_{j} v_{i}\right) \\
+\beta_{3} w_{j}\left(\nabla_{j} m_{i}-\nabla_{i} m_{j}\right)+\beta_{4} w_{j}\left(\nabla_{j} v_{i}-\nabla_{i} v_{j}\right) \\
-\beta_{5} h_{j}^{\Gamma} \nabla_{i} \Gamma_{j}+\beta_{6} \epsilon_{i j k} \nabla_{j} h_{k}^{\Gamma} \\
+\Gamma_{4} T_{k j i} \nabla_{j} \nabla_{k} T+\Gamma_{5} T_{i j k} \nabla_{j} \nabla_{k}(\Pi / \rho) \\
Y_{i}^{R}=\beta_{5} m_{j} \nabla_{j} \Gamma_{i}+\beta_{6} \epsilon_{i j k} \nabla_{j} m_{k}, \\
I_{\phi}^{R}=\gamma \nabla_{i} m_{i}+\beta_{7} A_{k k}+\left(\Gamma_{3} / \rho\right) T_{i j k} \nabla_{k} \nabla_{j} v_{i} \\
+\left(\Gamma_{5} / \rho\right) T_{i j k} \nabla_{k} \nabla_{j} m_{i},
\end{gathered}
$$

and

$$
j_{i}^{(h) R}=\gamma \rho m_{i}+\Gamma_{3} T_{i j k} \nabla_{j} v_{k}+\Gamma_{5} T_{i j k} \nabla_{j} m_{k}
$$

where we have kept nonlinear contributions when they contribute to the transport and convection of variables.

For the reversible coefficients in Eqs. (23)-(28) either sign is possible and no bounds can be given in general from the thermodynamic requirements associated with the second law of thermodynamics.

These reversible currents can be used to tune the transport and convective velocities of the different variables, cf. Sec. 6 of Ref. [13]. In particular, the $\beta_{1}$ term adds to the transport of the entropy density, Eq. (14). Since we assume that the mean velocity, $v_{i}$, is the appropriate transport velocity, we put $\beta_{1}=0$. In our model the transport velocity of $\dot{\rho}_{h}$ and $\dot{g}_{i}^{h}$ is $v_{i}^{h}$, while for $\dot{\rho}_{s}$ and $\dot{g}_{i}^{s}$ it is $v_{i}^{s}$. To achieve this we have to take $\gamma=0=\beta_{7}$, and $\beta_{2}=\beta_{4}=1 / 2$ and $\beta_{3}=1 / \rho_{h}-1 / \rho_{s}$, cf. Ref. [13]. Similarly, we assume that transport and convection of $\dot{\Gamma}$, Eq. (19), is done by the velocity $v_{i}^{h}$, which requires $\beta_{5}=2 \beta_{6}=(\rho \phi)^{-1}$.

Implementing these considerations Eqs. (23)-(28) simplify considerably to read

$$
\begin{gathered}
j_{i}^{(\sigma) R}=\Gamma_{2} T_{i j k} \nabla_{j} v_{k}+\Gamma_{4} T_{i j k} \nabla_{j} m_{k}, \\
\sigma_{i j}^{R}=m_{i} w_{j}-\Gamma_{2} T_{i j k} \nabla_{k} T \\
-\Gamma_{3} T_{i j k} \nabla_{k}(\Pi / \rho),
\end{gathered}
$$




$$
\begin{gathered}
X_{i}^{R}=w_{j} \nabla_{j} v_{i}+\left(\frac{1}{\rho_{h}}-\frac{1}{\rho_{s}}\right) w_{j}\left(\nabla_{j} m_{i}-\nabla_{i} m_{j}\right) \\
-\frac{1}{\rho \phi} h_{j}^{\Gamma} \nabla_{i} \Gamma_{j}+\frac{1}{2 \rho \phi} \epsilon_{i j k} \nabla_{j} h_{k}^{\Gamma} \\
+\Gamma_{4} T_{k j i} \nabla_{j} \nabla_{k} T+\Gamma_{5} T_{i j k} \nabla_{j} \nabla_{k}(\Pi / \rho) \\
Y_{i}^{R}=\frac{1}{\rho \phi} m_{j} \nabla_{j} \Gamma_{i}+\frac{1}{2 \rho \phi} \epsilon_{i j k} \nabla_{j} m_{k} \\
I_{\phi}^{R}=\left(\Gamma_{3} / \rho\right) T_{i j k} \nabla_{k} \nabla_{j} v_{i} \\
\quad+\left(\Gamma_{5} / \rho\right) T_{i j k} \nabla_{k} \nabla_{j} m_{i}
\end{gathered}
$$

and

$$
j_{i}^{(h) R}=\Gamma_{3} T_{i j k} \nabla_{j} v_{k}+\Gamma_{5} T_{i j k} \nabla_{j} m_{k},
$$

These equations will be also used in Sec. III and in Appendix A to discuss consequences of a novel dissipative cross-coupling as well as in Appendix B, where the effect of electric field gradients is elucidated.

The contributions $\sim \Gamma_{2 \ldots 5}$ are due to the existence of tetrahedral order and describe [29] how planar extensional flow (of the mean as well as of the relative velocity) give rise to reversible concentration and heat currents perpendicular to the plane. Vice versa, temperature and concentration gradients trigger shear stresses in the plane normal to the gradients, while suitable second order gradients lead to a temporal change of the relative velocity in the direction perpendicular to the gradients. We note that the latter contributions clearly represent a higher-order effect.

\section{E. Dissipative currents}

The dissipative parts of the currents introduced above can be deduced from a potential, the dissipation function $R$, that reads in bilinear approximation

$$
\begin{aligned}
2 R= & \zeta_{\Pi} \Pi^{2}+\xi m_{i} m_{i}+\kappa\left(\nabla_{i} T\right)\left(\nabla_{i} T\right)+\frac{1}{\gamma^{\Gamma}} h_{i}^{\Gamma} h_{i}^{\Gamma} \\
& +D\left(\nabla_{i} \Pi\right)\left(\nabla_{i} \Pi\right)+2 D^{(T)}\left(\nabla_{i} T\right)\left(\nabla_{i} \Pi\right) \\
& +v_{i j k l}\left(\nabla_{j} v_{i}\right)\left(\nabla_{l} v_{k}\right)+v_{i j k l}^{(w)}\left(\nabla_{j} m_{i}\right)\left(\nabla_{l} m_{k}\right) \\
& +2 v_{i j k l}^{(c)}\left(\nabla_{j} v_{i}\right)\left(\nabla_{l} m_{k}\right)+2 \Sigma T_{i j k} m_{i}\left(\nabla_{j} v_{k}\right)
\end{aligned}
$$

where the rank-4 viscositylike tensors have the same form as $K_{i j k l}$ in Eq. (6) containing three independent coefficientsone more than in the isotropic case $[32,47]$; explicitly,

$v_{i j k l}=v_{l} \delta_{i j} \delta_{k l}+\frac{v_{\text {tr }}}{2}\left(\delta_{i k} \delta_{j l}+\delta_{i l} \delta_{j k}-\frac{2}{3} \delta_{i j} \delta_{k l}\right)+v_{T} T_{j l p} T_{i k p}$.

The requirement of positivity of the entropy production associated with the second law of thermodynamics leads to a number of positivity requirements and bounds for the dissipative coefficients entering Eq. (35). All the diagonal coefficients must be positive leading to $\zeta_{\Pi}, \xi, \frac{1}{\gamma^{\Gamma}}, \kappa, D, v_{l}, v_{\mathrm{tr}}, v_{T}, v_{l}^{(w)}, v_{\mathrm{tr}}^{(w)}$, and $v_{T}^{(w)}$ all being positive. In addition, all cross-coupling terms associated with the nondiagonal coefficients are bounded from above by products of diagonal coefficients to guarantee positivity of the entropy production leading to the inequalities $\left(D^{(T)}\right)^{2}<\kappa D$, $\left(v_{l}^{(c)}\right)^{2}<v_{l} v_{l}^{(w)},\left(v_{\mathrm{tr}}^{(c)}\right)^{2}<v_{\mathrm{tr}} v_{\mathrm{tr}}^{(w)},\left(v_{T}^{(c)}\right)^{2}<v_{T} v_{T}^{(w)}$, and last, but not least $\Sigma^{2}<\xi \nu_{T}$. The influence of the cross-coupling coefficient $\Sigma$ will be elucidated in detail in the next section.

The last term of Eq. (35), $\sim \Sigma$, is specific for a two-fluid description of a fluid with isotropic and tetrahedral components. We note that it is of the same structure as the reversible coupling terms discussed in Ref. [29] between the density of linear momentum on one hand and temperature gradients, concentration gradients and electric fields on the other. The key difference is the different behavior of the relative velocity under time reversal.

As a consequence of this coupling, dissipative stresses are created in the plane perpendicular to a homogeneous relative velocity and, versa versa, an elongational flow can lead to a finite relative velocity in the stationary case. Further implications of this cross-coupling are discussed in Sec. III in detail.

From Eq. (35) the following dissipative currents are obtained,

$$
\begin{gathered}
j_{i}^{(\sigma) D}=-(\partial R) /\left(\partial \nabla_{i} T\right) \\
=-\kappa \nabla_{i} T-\rho \phi(1-\phi) d^{(T)} \nabla_{i} \Pi, \\
\sigma_{i j}^{D}=-(\partial R) /\left(\partial \nabla_{j} v_{i}\right) \\
=-v_{i j k l} \nabla_{l} v_{k}-v_{i j k l}^{(c)} \nabla_{l} m_{k}-\Sigma T_{i j k} m_{k}, \\
X_{i}^{D}=(\delta R) /\left(\delta m_{i}\right) \\
=\xi m_{i}+\Sigma T_{i j k}\left(\nabla_{j} v_{k}\right) \\
-\nabla_{j}\left(v_{i j k l}^{(w)} \nabla_{l} m_{k}+v_{k l i j}^{(c)} \nabla_{l} v_{k}\right), \\
I_{\phi}^{D}=(\delta R) /(\delta \Pi) \\
=\zeta_{\Pi} \Pi-\rho d \nabla_{i}^{2} \Pi-\rho \phi(1-\phi) d^{(T)} \nabla_{i}^{2} T \\
Y_{i}^{D}=(\partial R) /\left(\partial h_{i}^{\Gamma}\right) \\
=\frac{1}{\gamma^{\Gamma}} h_{i}^{\Gamma} .
\end{gathered}
$$

The relative velocity, $w_{i}$, always relaxes, since it is not related to any spontaneously broken continuous symmetry, nor to a conservation law. The tetrahedral orientation is diffusive, since $h_{i}^{\Gamma} \sim \nabla_{j} \nabla_{k} \Gamma_{k}$ according to Eq. (5). Only in the case of an external orienting field, $h_{i}^{\Gamma}$ is homogeneous and $\Gamma_{i}$ relaxes (toward a preferred field direction). Diffusion and thermodiffusion is written in the usual way with $D=\rho d$ and $D^{(T)}=\alpha d^{(T)}$. For a conserved density, $\rho_{h}$, the relaxation coefficient $\zeta_{\Pi}$ is zero (meaning the relaxation time goes to infinity), and the linearized concentration current has the standard form of a binary mixture:

$$
j_{i}^{(\phi) D}=-\rho D \nabla_{i}(\Pi / \rho)-\rho D^{(T)} \nabla_{i} T .
$$

\section{CONSEQUENCES OF THE DISSIPATIVE CROSS-COUPLING $\Sigma$}

In this section we analyze in some detail the consequences of the dissipative cross-coupling term, $\Sigma$, between velocity gradients and the relative velocity, $w_{i}$, introduced in Eq. (35). 
To be specific we consider a sample under the influence of a planar shear flow,

$$
S=\nabla_{x} v_{y}
$$

The tetrahedral $T_{d}$ phase is characterized by the existence of the octupolar order parameter $T \equiv T_{i j k}=\Sigma_{\alpha=1}^{4} n_{i}^{\alpha} n_{j}^{\alpha} n_{k}^{\alpha}$ expressed by the four unit vectors, $\mathbf{n}^{\alpha}$, with $\alpha=1,2,3$ and 4 defining a tetrahedron [26,32]. $T$ is fully symmetric in all three indices and odd under parity, since the $n^{\alpha}$ 's are vectors, or, in physical terms, are polar. The orientation of the tetrahedron in laboratory space is arbitrary, but the matrix representation [26]

$$
T_{i j k}=\frac{T_{0}}{\sqrt{3}}\left(\begin{array}{cccc}
1 & 1 & -1 & -1 \\
1 & -1 & 1 & -1 \\
-1 & 1 & 1 & -1
\end{array}\right)
$$

is very convenient for the following investigation. The spatially inverted system, where all signs in Eq. (44) are reversed, describes an orientation that is different from the original one, but energetically equivalent.

In lowest order the linearized macroscopic equations for the concentration $\phi$ and the velocity difference $w_{i}$ take the form making use of Eq. (44)

$$
\begin{gathered}
\dot{\phi}+\frac{\alpha}{\rho}\left(\nabla_{x} w_{x}+\nabla_{y} w_{y}+\nabla_{z} w_{z}\right)+\frac{\zeta_{\Pi}}{\kappa_{\phi}} \delta \phi=0, \\
\dot{w}_{x}+\frac{1}{\kappa_{\phi} \rho} \nabla_{x} \phi+\alpha \xi w_{x}=0, \\
\dot{w}_{y}+\frac{1}{\kappa_{\phi} \rho} \nabla_{y} \phi+\alpha \xi w_{y}+w_{x} S=0, \\
\dot{w}_{z}+\frac{1}{\kappa_{\phi} \rho} \nabla_{z} \phi+\alpha \xi w_{z}+\frac{4}{3 \sqrt{3}} T_{0} \Sigma S=0 .
\end{gathered}
$$

In a next step we look for an exact homogeneous solution of Eqs. (45)-(48) and obtain

$$
\delta \phi_{0}=w_{x}^{(0)}=w_{y}^{(0)}=0,
$$

while

$$
\dot{w}_{z}+\alpha \xi w_{z}+\frac{4}{3 \sqrt{3}} T_{0} \Sigma S=0,
$$

leading, for a time-periodic shear rate $S=S_{0} \cos (\Omega t)$, to

$$
w_{z}^{o s c}(t)=-\frac{4}{3 \sqrt{3}} T_{0} \Sigma S_{0} \frac{\cos (\Omega t+\chi)}{\sqrt{\Omega^{2}+\xi^{2} \alpha^{2}}}+w_{z}^{0} \exp (-\alpha \xi t),
$$

where $w_{z}^{0}$ is a constant determined by an initial condition. In the long-time limit $t \gg 1 / \alpha \xi$, an oscillation with a phase-shift $\chi=\arctan (\Omega / \alpha \xi)$ is obtained.

In the limit $\Omega \rightarrow 0$ a stationary solution is found,

$$
w_{z}^{\text {stat }}(t \rightarrow \infty)=-\frac{4}{3 \sqrt{3}} T_{0} \Sigma S_{0} \frac{1}{\xi \alpha} .
$$

This result has a simple interpretation: If one applies a shear in the $x-y$ plane, an oscillation of the relative velocity in the $z$ direction, perpendicular to the shear plane results. Thus, the presence of the tetrahedral or octupolar order parameter immediately renders the flow three-dimensional.
To investigate which spatial excitations might exist as a function of the $z$ direction, we look for excitations $w_{z}(\omega, z)$ and $\phi(\omega, z)$ with excitation frequency $\omega$ and fixed wave vector $k_{z}$.

With the ansatz

$$
\left(\phi, w_{z}\right)=\left(\Phi, W_{z}\right) \exp \left(i \omega t+i k_{z} z\right)
$$

and with $w_{x}=w_{y}=0$, Eqs. (45)-(48) give rise to the dispersion relation

$$
\omega^{2}-i \omega\left(\frac{\zeta_{\pi}}{\kappa_{\phi}}+\alpha \xi\right)-k_{z}^{2} \frac{\alpha}{\kappa_{\phi} \rho^{2}}-\frac{\zeta_{\pi} \alpha \xi}{\kappa_{\phi}}=0
$$

with the solution

$$
2 \omega=i\left(\frac{\zeta_{\pi}}{\kappa_{\phi}}+\alpha \xi\right) \pm \sqrt{4 k_{z}^{2} \frac{\alpha}{\kappa_{\phi} \rho^{2}}-\left(\frac{\zeta_{\pi}}{\kappa_{\phi}}-\alpha \xi\right)^{2}} .
$$

Equation (55) contains a number of interesting special cases. First of all we notice that in the limit $\zeta_{\pi} \rightarrow 0$ and $\xi \rightarrow 0$ (meaning that $\phi$ and $w_{i}$ both do not relax) one obtains a propagating mode coupling concentration $\phi$ and relative velocity $w_{z}$ in the direction perpendicular to the shear plane

$$
\omega= \pm k_{z} \sqrt{\frac{\alpha}{\kappa_{\phi} \rho^{2}}},
$$

with velocity $c^{2}=\alpha /\left(\kappa_{\phi} \rho^{2}\right)$. This would correspond to a sound like mode coupling concentrations and $w_{z}$. It would be a situation reminiscent to second sound in a superfluid coupling the superfluid velocity, $v_{s}$ to entropy density, $\sigma$. However, as discussed in Sec. II, the velocity difference is not conserved, but is a macroscopic variable, which relaxes on sufficiently long, but finite time. Thus even for a concentration variable that is conserved, an overdamped mode in $z$ direction results, which reads in leading order in $k_{z}$,

$$
2 \omega=i \alpha \xi \pm \sqrt{4 k_{z}^{2} \frac{\alpha}{\kappa_{\phi} \rho^{2}}-(\alpha \xi)^{2}} .
$$

If $\xi$ is sufficiently small, then we expect that an experiment will show a finite regime in $k_{z}$, for which this mode propagates.

In case also the concentration $\phi$ relaxes, the dispersion relation is given by Eq. (55) reflecting the presence of both relaxation channels in the long wavelength limit.

\section{CONCLUSIONS}

In this paper we have studied the changes of the macroscopic behavior in two-fluid systems, when one of the two fluids is of tetrahedral-octupolar nature leading to broken parity symmetry. For simplicity we have focused on optically isotropic systems. The existence of tetrahedral order has been shown to lead to a number of reversible and irreversible crosscoupling terms to the relative velocity, which do not exist without octupolar order. Indeed, it turns out that tetrahedral order has a profound influence on the dynamics of two-fluid systems. In particular it emerges that a dissipative crosscoupling between velocity gradients and the relative velocity can lead to a relative velocity in the direction perpendicular to an applied planar shear flow. In this geometry one can 
then excite an overdamped propagating mode in this direction formed by the concentration variations and the relative velocity. In Appendix A we have shown that there is, in addition, a reversible cross-coupling between the relative velocity and electric gradient fields. The structure of this reversible cross-coupling is isomorphic to the dissipative cross-coupling of $w_{i}$ to velocity gradients. Thus one can use two simple external forces, namely, a planar shear flow and a planar electric gradient field, to study experimentally the physical consequences of two cross-coupling terms characteristic for the two-fluid behavior in a system with octupolar-tetrahedral order, one dissipative and one reversible. In Appendix B we have presented a reversible coupling of the relative velocity to rotations of the tetrahedral orientation in a chiral phase.

\section{APPENDIX A: CONSEQUENCES OF THE DISSIPATIVE CROSS-COUPLING $\Sigma$ IN THE PRESENCE OF AN EXTENSIONAL FLOW}

In this Appendix we study the influence of the dissipative cross-coupling term, $\Sigma$, between velocity gradients and the relative velocity, $w_{i}$, introduced in Eq. (35), in the presence of an extensional flow.

To be specific we consider a sample under the influence of two types of extensional flow, namely, parallel and perpendicular to a preferred direction, which we take to be the $\hat{z}$ direction,

$$
\begin{aligned}
& E^{(1)}=\nabla_{z} v_{z}, \\
& E^{(2)}=\nabla_{x} v_{x} .
\end{aligned}
$$

We take the orientation of the tetrahedral order to be parallel to one of the tetrahedral directions, which can be achieved by an external electric field. In this case we have for the matrix representation of $T_{i j k}[30,32]$

$$
T_{i j k}=\frac{T_{0}}{3}\left(\begin{array}{cccc}
0 & -\sqrt{2} & -\sqrt{2} & 2 \sqrt{2} \\
0 & -\sqrt{6} & \sqrt{6} & 0 \\
3 & -1 & -1 & -1
\end{array}\right)
$$

which is appropriate for the following investigation.

The spatially inverted system, where all signs in Eq. (A3) are reversed, describes an orientation that is different from the original one, but energetically equivalent.

In lowest order the linearized macroscopic equations for the concentration $\phi$ and the velocity difference $w_{i}$ take the form making use of Eq. (A3)

$$
\begin{gathered}
\dot{\phi}+\frac{\alpha}{\rho}\left(\nabla_{x} w_{x}+\nabla_{y} w_{y}+\nabla_{z} w_{z}\right)+\frac{\zeta \Pi}{\kappa_{\phi}} \delta \phi=0 \\
\dot{w}_{i}+\frac{1}{\kappa_{\phi} \rho} \nabla_{i} \phi+\alpha \xi w_{i}+w_{j} \nabla_{j} v_{i}+\Sigma T_{i j k} \nabla_{j} v_{k}=0
\end{gathered}
$$

Now we are prepared to discuss the two cases of Eqs. (A1) and (A2) separately. For the case of an extensional flow in $\hat{z}$ direction we have

$$
\begin{gathered}
\dot{\phi}+\frac{\alpha}{\rho}\left(\nabla_{x} w_{x}+\nabla_{y} w_{y}+\nabla_{z} w_{z}\right)+\frac{\zeta_{\Pi}}{\kappa_{\phi}} \delta \phi=0, \\
\dot{w}_{x}+\frac{1}{\kappa_{\phi} \rho} \nabla_{x} \phi+\alpha \xi w_{x}=0
\end{gathered}
$$

$$
\begin{gathered}
\dot{w}_{y}+\frac{1}{\kappa_{\phi} \rho} \nabla_{y} \phi+\alpha \xi w_{y}=0 \\
\dot{w}_{z}+\frac{1}{\kappa_{\phi} \rho} \nabla_{z} \phi+\alpha \xi w_{z}+\frac{8}{9} T_{0} \Sigma E^{(1)}+w_{z} E^{(1)}=0 .
\end{gathered}
$$

Comparing Eqs. (A6)-(A9) with the case of an external shear flow Eqs. (45)-(48), we read off immediately, that only the equation for $w_{z}$, Eq. (A9), differs from the case of shear flow: instead of $\frac{4}{3 \sqrt{3}} T_{0} \Sigma S$ we have now $\frac{8}{9} T_{0} \Sigma E^{(1)}+w_{z} E^{(1)}$. Thus, the driving force for elongations parallel to the preferred $\hat{z}$ direction not only enters as an inhomogeneity, but also via $w_{z} E^{(1)}$.

To examine similarities and differences to the case of an applied shear flow, we proceed as follows. We first look for an exact homogeneous solution of Eqs. (A6)-(A9) and use

$$
\delta \phi_{0}=w_{x}^{(0)}=w_{y}^{(0)}=0,
$$

while

$$
\dot{w}_{z}+\alpha \xi w_{z}+E^{(1)} w_{z}+\frac{8}{9} T_{0} \Sigma E^{(1)}=0 .
$$

As for an applied shear flow, we can look again at a timedependent forcing $\sim E^{(1)}=E_{0}^{(1)} \cos (\Omega t)$ leading, in the limit of vanishing frequency $\Omega$, to a stationary solution

$$
w_{z}^{\text {stat }}(t \rightarrow \infty)=-\frac{8}{9} \Sigma T_{0} E_{0}^{(1)} \frac{1}{\xi \alpha+E_{0}^{(1)}} .
$$

This result has a straightforward interpretation: applying an extensional flow parallel to the preferred $\hat{z}$ direction, a spatially homogeneous oscillation of the relative velocity in the $\hat{z}$-direction results. We note that-in contrast to the case of an external shear flow, Eq. (52) — the amplitude of $w_{z}$ is not linear in the driving force and saturates to a finite value for strong driving $E_{0}^{(1)} \rightarrow \infty$.

However, it is well known that parametric driving, like the term $E^{(1)} w_{z}$, can lead to an instability of the stationary solution giving rise to subharmonic temporal modes. A detailed study of such instabilities is beyond the scope of this manuscript. The effect is specific for extensional flow and does not arise for shear flow.

To investigate spatial excitations as a function of the $z$ direction, we proceed in parallel to the analysis given in Sec. III: We look for excitations $w_{z}(\omega, z)$ and $\phi(\omega, z)$ with excitation frequency $\omega$ and fixed wave vector $k_{z}$. With the ansatz

$$
\left(\phi, w_{z}\right)=\left(\Phi, W_{z}\right) \exp \left(i \omega t+i k_{z} z\right)
$$

and with $w_{x}=w_{y}=0$, Eqs. (A6)-(A13) give rise to the modified dispersion relation

$$
\omega^{2}-i \omega\left(\frac{\zeta_{\pi}}{\kappa_{\phi}}+\alpha \xi+E_{0}^{(1)}\right)-k_{z}^{2} \frac{\alpha}{\kappa_{\phi} \rho^{2}}-\frac{\zeta_{\pi}}{\kappa_{\phi}}\left(\alpha \xi+E_{0}^{(1)}\right)=0,
$$

signalling the fact that now the excitation frequency of the dispersion relation depends on the amplitude of the driving field, $E_{0}^{(1)}$.

Comparing the present dispersion relation with the case of an applied shear flow, Eq. (54), we see that most of the discussion of Sec. III can be carried over to the present case of an 
extensional flow parallel to the preferred direction, provided $\alpha \xi$ is replaced by $\alpha \xi+E_{0}^{(1)}$, and some care is taken regarding the sign under the square root in the dispersion relation.

For the second case of a extensional flow in the $x$ direction, perpendicular to the preferred $\hat{z}$ direction, we find

$$
\begin{gathered}
\dot{\phi}+\frac{\alpha}{\rho}\left(\nabla_{x} w_{x}+\nabla_{y} w_{y}+\nabla_{z} w_{z}\right)+\frac{\zeta_{\Pi}}{\kappa_{\phi}} \delta \phi=0, \\
\dot{w}_{x}+\frac{1}{\kappa_{\phi} \rho} \nabla_{x} \phi+\alpha \xi w_{x} \\
+\frac{4 \sqrt{2}}{9} T_{0} \Sigma E^{(2)}+w_{x} E^{(2)}=0 \\
\dot{w}_{y}+\frac{1}{\kappa_{\phi} \rho} \nabla_{y} \phi+\alpha \xi w_{y}=0 \\
\dot{w}_{z}+\frac{1}{\kappa_{\phi} \rho} \nabla_{z} \phi+\alpha \xi w_{z}-\frac{4}{9} T_{0} \Sigma E^{(2)}=0 .
\end{gathered}
$$

This case is similar to the previous case, Eqs. (A6)-(A9), regarding the parametric driving, $\sim E^{(2)} w_{x}$, that occurs along the external flow, but is different with respect to the inhomogeneous driving, $\sim \Sigma E^{(2)}$, that takes place in both, the external flow direction and the preferred direction $(z)$. Proceeding as before we find a stationary solution for $w_{x}$ and $w_{z}$ of the following form:

$$
\begin{gathered}
w_{x}^{\text {stat }}(t \rightarrow \infty)=-\frac{4 \sqrt{2}}{9} \Sigma T_{0} E_{0}^{(2)} \frac{1}{\xi \alpha+E_{0}^{(2)}}, \\
w_{z}^{\text {stat }}(t \rightarrow \infty)=\frac{4}{9} \Sigma T_{0} E_{0}^{(2)} \frac{1}{\xi \alpha} .
\end{gathered}
$$

Analyzing spatial excitations in both, the external flow and the preferred direction, described by finite $k_{x}$ and $k_{z}$ simultaneously, one obtains three coupled equations for the three variables $w_{x}, w_{z}$, and $\phi$ along with a cubic dispersion relation. In this case only $w_{y}$ is decoupled.

\section{APPENDIX B: DYNAMIC EFFECTS RELATED TO ELECTRIC FIELDS AND THEIR GRADIENTS}

For the dissipative effects of electric fields we have in the dissipation function the contributions

$$
2 R^{(e)}=\sigma^{(e)} E_{i} E_{i}+2 D^{(e \phi)}\left(\nabla_{i} \Pi\right) E_{i}+2 D^{(e T)}\left(\nabla_{i} T\right) E_{i} .
$$

This is the standard form for an isotropic system of a miscible mixture with the electric conductivity, $\sigma^{(e)}$, the thermoelectric effect, $D^{(e T)}$, and the cross-coupling between electric fields and the thermodynamic conjugate of the concentration, $D^{(e \phi)}$. Positivity of the entropy production requires $\sigma^{(e)}>0$, $\left(D^{(e \phi)}\right)^{2}<\sigma^{(e)} D$, and $\left(D^{(e T)}\right)^{2}<\sigma^{(e)} \kappa$. There is no dissipative cross-coupling between electric fields on the one hand, and gradients of the barycentric velocity field, $A_{i j}$, and the velocity difference, $w_{i}$, on the other, because of time reversal symmetry:

$$
\begin{gathered}
j_{i}^{(e) R}=\beta_{1}^{(e)} m_{i}+\Gamma_{1} T_{i j k} \nabla_{j} v_{k}+\Gamma_{6} T_{i j k} \nabla_{j} m_{k}, \\
\sigma_{i j}^{(e), R}=-\Gamma_{1} T_{i j k} E_{k}, \\
X_{i}^{(e), R}=\beta_{1}^{(e)} E_{i}+\Gamma_{6} T_{k j i} \nabla_{j} E_{k} .
\end{gathered}
$$

Since we assume that the mean velocity, $v_{i}$, is the appropriate transport velocity, we put $\beta_{1}^{(e)}=0$. The terms $\sim \Gamma_{1}$ have been given in Ref. [29] including a discussion of their physical implications. The cross-coupling $\sim \Gamma_{6}$ represents a new reversible coupling between the electric currents and gradients of the relative velocity field on one hand and between the reversible current associated with relative velocities and gradients of an electric field. It can only exist because of the presence of octupolar order and is unknown from the other two-fluid systems studied so far. Inspecting its structure in detail it emerges that it is isomorphic to the dissipative coupling between the relative velocity, $w_{i}$, and symmetric velocity gradients, $A_{i j}$. The key difference, reversible versus dissipative, can be traced back to the different behavior under time reversal for the electric field versus the field of barycentric velocity. Since the contributions given in Eqs. (B2)-(B4) are reversible in nature, the coefficients $\Gamma_{1}$ and $\Gamma_{6}$ can be of either sign.

This also indicates a possibility to perform controlled experiments on this cross-coupling term $\sim \Gamma_{6}$. Instead of applying a shear flow as in Sec. III, one can apply a planar gradient in the electric field. This in turn will then lead to a temporally varying relative velocity $w_{i}$. The analog of Eq. (48) reads

$$
\dot{w}_{z}+\frac{1}{\kappa_{\phi} \rho} \nabla_{z} \phi+\alpha \xi w_{z}+\frac{4}{3 \sqrt{3}} T_{0} \Gamma_{6} \nabla_{y} E_{x}=0,
$$

where we have taken the same coordinate system as in Sec. III.

\section{APPENDIX C: REVERSIBLE COUPLING OF THE RELATIVE VELOCITY TO TETRAHEDRAL ORDER IN THE CHIRAL T PHASE}

In the bulk of this manuscript we have given a two-fluid macroscopic dynamic description for a two-fluid system for which one component shows tetrahedral order of $T_{d}$ symmetry, which is nonchiral. In this short Appendix we briefly discuss how the relative velocity, $w_{i}$, can couple to the other macroscopic variables in the $T$ phase, which is chiral and of lower symmetry than the $T_{d}$ phase. The chirality can be characterized by a pseudoscalar $q_{0}$ in the same spirit as in a cholesteric phase [8,35]. The chiral $T$ phase and some of its physical properties have been studied by Fel [26] and the macroscopic dynamics of the $T$ phase has been given in Refs. [31,32]. There it has been shown that there is a reversible coupling between the macroscopic variables associated with tetrahedral order, $\delta \Gamma_{i}$, and the rate of strain tensor, $A_{i j}$, via the equation [31,32]

$$
Y_{i}^{R}=\cdots+q_{0} \lambda T_{i j k} A_{j k}
$$

Together with rotational flow this coupling leads to flow alignment for a stationary situation in an optically isotropic system (compare Refs. [31,32] for a detailed discussion).

Here we point out that in the chiral $T$ phase as part of a two-fluid system there is an analogous reversible coupling between the relative velocity, $w_{i}$, and the dynamics of rotations given by

$$
\dot{\Gamma}_{i}+Y_{i}=0
$$


As a result we obtain

$$
\begin{aligned}
X_{i}^{R} & =\cdots+q_{0} \lambda_{w} T_{i j k} \nabla_{j} h_{k}^{\Gamma}, \\
Y_{i}^{R} & =\cdots+q_{0} \lambda_{w} T_{i j k} \nabla_{j} m_{k} .
\end{aligned}
$$

Equations (C3) and (C4) clearly demonstrate the close resemblance to the flow alignment term found for the $T$ phase in Refs. [31,32]. The contribution $\sim \lambda_{w}$ in both equations also shows that the dynamics of rotations is significantly influ- enced by a coupling to gradients of the relative velocity. We note that due to this additional coupling $\sim \lambda_{w}$ the rotational dynamics of $\delta \Gamma_{i}$ will be more complex compared to a $T$ phase in a one component fluid, since both, gradients of the velocity field and gradients of the relative velocity [via $\sim \beta_{6}=$ $1 /(2 \rho \phi)$ from Eq. (26)] and $\sim \lambda_{w}$ enter the picture. Both being associated with a reversible coupling, the coefficients $\lambda$ and $\lambda_{w}$ could be of either sign from the point of view of thermodynamics. Since the structure of the coupling terms involving $\lambda$ and $\lambda_{w}$ is the same, one might speculate that they are similar in magnitude.
[1] L. D. Landau and E. M. Lifshitz, Hydrodynamics (Pergamon Press, New York, 1982).

[2] P. C. Martin, O. Parodi, and P. Pershan, Phys. Rev. A 6, 2401 (1972).

[3] D. Forster, Hydrodynamic Fluctuations, Broken Symmetry, and Correlation Functions (Benjamin, Reading, MA, 1975).

[4] I. M. Khalatnikov, Introduction to the Theory of Superfluidity (W. A. Benjamin, Reading, MA, 1965).

[5] P. C. Hohenberg and P. C. Martin, Ann. Phys. (NY) 34, 291 (1965).

[6] R. Graham, Phys. Rev. Lett. 33, 1431 (1974).

[7] D. Forster, Ann. Phys. (NY) 84, 505 (1974).

[8] P. G. De Gennes, The Physics of Liquid Crystals (Clarendon Press, Oxford, 1975).

[9] H. Pleiner and H. R. Brand, in Pattern Formation in Liquid Crystals, edited by A. Buka and L. Kramer (Springer, New York, 1996).

[10] P. Hebraud, F. Lequeux, and J. F. Palierne, Langmuir 16, 8296 (2000).

[11] D. Lhuillier, J. Non-Newtonian Fluid Mech. 96, 19 (2001).

[12] R. G. Larson, Constitutive Equations for Polymer Melts and Solutions (Butterworths, Boston, MA, 1988).

[13] H. Pleiner and J. L. Harden, Notices of Universities. South of Russia. Natural Sciences, special issue: Nonlinear Problems of Continuum Mechanics (2003), pp. 46-61; retrieved from arXiv:cond-mat/0404134.

[14] H. Pleiner and J. L. Harden, AIP Proc. 708, 46 (2004).

[15] R. Graham and H. Pleiner, Phys. Rev. Lett. 34, 792 (1975).

[16] M. Liu, Phys. Rev. B 13, 4174 (1976).

[17] R. Graham and H. Pleiner, J. Phys. C 9, 279 (1976).

[18] H. R. Brand, M. Dörfle, and R. Graham, Ann. Phys. (NY) 119, 434 (1979).

[19] M. Liu, Phys. Rev. Lett. 43, 1740 (1979).

[20] H. R. Brand and H. Pleiner, Phys. Rev. B 102, 094510 (2020).

[21] H. R. Brand and H. Pleiner, Phys. Rev. E 103, 012705 (2021).

[22] H. Pleiner, D. Svenšek, T. Potisk, and H. R. Brand, Phys. Rev. E 101, 032601 (2020).

[23] H. Pleiner, A. M. Menzel, and H. R. Brand, Phys. Rev. B 103, 174304 (2021).
[24] H. Pleiner and H. R. Brand, Rheol. Acta 60, 675 (2021).

[25] M. Tinkham, Group Theory and Quantum Mechanics (McGraw-Hill, New York, 1964).

[26] L. Fel, Phys. Rev. E 52, 702 (1995).

[27] L. Radzihovsky and T. C. Lubensky, Europhys. Lett. 54, 206 (2001).

[28] T. C. Lubensky and L. Radzihovsky, Phys. Rev. E 66, 031704 (2002).

[29] H. R. Brand, H. Pleiner, and P. E. Cladis, Eur. Phys. J. E 7, 163 (2002).

[30] H. R. Brand and H. Pleiner, Eur. Phys. J. E 31, 37 (2010).

[31] H. Pleiner and H. R. Brand, Eur. Phys. J. E 37, 11 (2014).

[32] H. Pleiner and H. R. Brand, Braz. J. Phys. 46, 565 (2017).

[33] T. Ostapenko, D. B. Wiant, S. N. Sprunt, A. Jakli, and J. T. Gleeson, Phys. Rev. Lett. 101, 247801 (2008).

[34] D. Wiant, K. Neupane, S. Sharma, J. T. Gleeson, S. Sprunt, A. Jakli, N. Pradhan, and G. Iannacchione, Phys. Rev. E 77, 061701 (2008).

[35] P. G. de Gennes, Mol. Cryst. Liq. Cryst. 12, 193 (1971).

[36] B. Mettout, Phys. Rev. E 74, 041701 (2006).

[37] L. Longa, G. Pajak, and T. Wydro, Phys. Rev. E 79, 040701(R) (2009).

[38] K. Trojanowski, G. Pajak, L. Longa, and T. Wydro, Phys. Rev. E 86, 011704 (2012).

[39] L. Longa and K. Trojanowski, Acta. Phys. Pol. B 44, 1201 (2013).

[40] E. G. Virga, Eur. Phys. J. E 38, 63 (2015).

[41] G. Gaeta and E. G. Virga, Eur. Phys. J. E 39, 113 (2016).

[42] Y. Chen, L. Qi, and E. G. Virga, J. Phys. A: Math. Theor. 51, 025206 (2018).

[43] T. Ohta, T. Ohkuma, and K. Shitara, Phys. Rev. E 80, 056203 (2009).

[44] M. Tarama and T. Ohta, Phys. Rev. E 87, 062912 (2013).

[45] T. Hiraiwa, M. Y. Matsuo, T. Okhuma, T. Ohta, and M. Sano, Europhys. Lett. 91, 20001 (2010).

[46] H. B. Callen, Thermodynamics and an Introduction to Thermostatics (John Wiley \& Sons, New York, 1985).

[47] W. P. Mason, Physical Acoustics and the Properties of Solids (D. Van Nostrand, New York, 1958). 